\title{
Finger Knuckle Print Identification using Gabor Features
}

\author{
Shubhangi Neware \\ Research Scholar \\ Dr. C.V. Raman University \\ Bilaspur (C.G)
}

\author{
Kamal Mehta, Ph.D \\ Associate Professor \\ Institute of Technology, Nirma \\ University, Ahmadabad (Gujrat)
}

\author{
A. S. Zadgaonkar, Ph.D \\ Advisor \\ Dr. C.V. Raman University \\ Bilaspur (C.G)
}

\begin{abstract}
One of the current trends in biometric human identification is the development of new emerging modalities. Knuckle biometrics is one of such promising modalities. Texture pattern produced by the finger knuckle bending is highly unique and makes the surface a distinctive biometric identifier. This paper presents feature based identification methods for an emerging biometric identifier called FingerKnuckle-Print (FKP). Techniques employed for feature based approach is Gabor filter method .In applications of computer vision and image analysis, Gabor filters have maintained their popularity in feature extraction for almost three decades. In the proposed work experiment is carried out to identify finger knuckle images of more than 100 persons. Compared with the other existing finger back surface based biometric system, the proposed FKP system achieves much higher recognition rate.
\end{abstract}

\section{General Terms}

Biometrics, Gabor filter, Personal authentication

\section{Keywords}

Finger-Knuckle-Print (FKP)

\section{INTRODUCTION}

Personal identification and authentication is a common concern to both industries and academic research due to its various applications. Biometrics is the discipline of recognizing a person's identity based on his/her physical or behavioural characteristics or traits. In the last three decades, researchers have exhaustively investigated the use of a number of biometric characteristics. Biometrics has attracted much attention in the recent decade due to its numerous applications in various fields. There are various biometric identifiers used by different researchers such as face, fingerprint, iris, signature, voice, palm print etc. Each of this traditional biometric identifier has its own advantages and disadvantages. Researchers have broad scope in searching new kinds of biometric identifiers. Hand-based biometrics has attracted lot of attention for personal identification by using palm print, hand geometry, 3-D finger geometry and hand vein. But these systems have certain drawbacks. Palm print recognition systems have not yet been deployed for civilian applications (e.g., access control), because of its large physical size. The hand geometry systems also have large physical size, so they cannot be easily embedded in existing security systems. The pattern of blood vessels hidden underneath the skin is quite distinct in individuals, even among identical twins and stable over long period of time. But this could not be primarily used because it is very expensive system compared to other biometric systems. Also it is not adopted due to lack of large scale studies on vein individuality and stability [1]. Although fingerprint-based recognition has been the longest serving, most successful and popular method for person identification, it has some hygienic issues. Touching the finger to the same scanner repetitively by many users may cause skin problems. This problem may occur with other biometric system that uses touched sensors. Also the earlier approaches achieved the results by constraining the position and posture of hands by using pegs. However, such approaches are inconvenient and difficult for some user groups, especially for the children and old persons. Therefore, a lot of emphasis has been laid on contact free and peg-free image acquisition .The peg-free imaging, although highly convenient to users, generates images with more scale and rotational variations. Therefore, efforts are further required to get better results and to improve the reported performance [2].

The finger back surface, also known as the dorsum of the hand, can be highly useful in user identification and has not yet attracted the attention of researchers. The image-pattern formed from the finger-knuckle bending is highly unique and makes this finger knuckle surface a distinctive biometric identifier. Finger geometry features can be acquired from the same image, at the same time, and integrated to improve the performance of the system. The peg-free imaging of the finger back surface is highly convenient and user friendly. Such images can be acquired online and used to extract scale, translation, and rotational-invariant knuckle features for user identification. The contact free imaging of the finger back surface is highly beneficial to users [2]. It is reported that the skin pattern on the finger-knuckle is highly rich in texture due to skin folds and creases, and hence, can be considered as a biometric identifier. Various advantages of using Finger Knuckle Print (FKP) include rich in texture features, easily accessible, contact-less image acquisition, invariant to emotions and other behavioral aspects such as tiredness, stable features and acceptability in the society [3]. Literature survey of related work has already been discussed in detail, in our earlier papers [11] [24].

\section{PROPOSED METHODOLOGY}

Proposed work uses 2D Gabor filters to extract the image local orientation information. This information is then employed to extract and represent the FKP features. While matching of two knuckles, the angular distance is used to measure the similarity.

\subsection{Feature Extraction}

The Gabor filtering technique can simultaneously extract the spatial-frequency information from the original signal [4]. It has been widely used as a convolution filter to fulfill the feature extraction job. Since 1980s, it has been widely used as an effective tool to fulfill the feature extraction job in face, iris, and fingerprint and palm print recognition systems. In [5], 
Loris and Alessandra described a Gabor feature selection technique. The Gabor filter can produce three types of features that are phase, magnitude and orientation, which can be used separately or jointly in different systems. Recently, Kong evaluated the three basic features (magnitude, phase, and orientation) produced by the Gabor filter for face recognition and concludes that the orientation feature is the most robust and distinctive feature [6]. T.S. Lee [8] derive the conditions under which a set of continuous 2D Gabor wavelets will provide a complete representation of any image, and also find self-similar wavelet parameterizations which allow stable reconstruction by summation as though the wavelets formed an orthonormal basis. In this paper, we proposed a method combining the orientation and magnitude features using Gabor filter for FKP recognition. Experimental results in section 3 verify that the proposed scheme gives better performance in FKP recognition than the other codingbased methods. The Gabor function has different forms in the literature and here we adopt the one of the form proposed by Lee [8] as given below:

$$
\begin{aligned}
& G(x, y, \omega, \theta) \\
& =\frac{\omega}{\sqrt{2} \pi k} e^{-\frac{\omega^{2}}{8 k^{2}}\left(4 x^{\prime}{ }^{2}+y^{\prime}\right)}\left[e^{i \omega x^{\prime}}-e^{-k^{2} / 2}\right]
\end{aligned}
$$

Where

$\mathrm{x}^{\prime}=(x-x 0) \cos \theta+(y-y 0) \sin \theta$,

$y^{\prime}=-(x-x 0) \sin \theta+(y-y 0) \cos \theta$,

$(\mathrm{x} 0, y 0)$ is the center of the function, $\omega$ is the radial frequency in radians per unit length and $\theta$ is the orientation of the Gabor functions in radians. $\mathrm{k}$ is defined by $k=\sqrt{2} \ln 2\left(\frac{2^{\delta}+1}{2^{\delta}-1}\right)$ , where $\delta$ is the half-amplitude bandwidth of the frequency response. $\omega$ can be determined by $\omega=\frac{k}{\sigma}$., where $\sigma$ is the standard deviation of the Gaussian envelop. The 2D filter represented by $G(x, y, \omega, \theta)$ extracts the orientation and magnitude information at each pixel in digital knuckle image.

\subsection{FKP Matching}

Degree of similarity can be determined by calculating angular distance between two feature matrices. Let $\mathrm{P}$ and $\mathrm{Q}$ be the two feature matrices, and $\mathrm{Pm}$ and $\mathrm{Qm}$ be corresponding masks used for indicating the overlapping areas when one of the feature is translated. Then the angular distance $\mathrm{D}(\mathrm{P}, \mathrm{Q})$ is defined by following equation:

$\mathrm{D}(\mathrm{P}, \mathrm{Q})=$

$$
\begin{aligned}
\sum_{y=1}^{\text {rows cols }} \sum_{x=1}((P m(x, y) & \cap Q m(x, y)) \\
\times & G(P(x, y), Q(x, y)) \\
& / 3 \sum_{y=1}^{\text {rows col }} \sum_{x=1}^{\operatorname{Pm}(x, y) \cap Q m(x, y)}
\end{aligned}
$$

Where $\mathrm{n}$ denotes the logical AND operator and

$$
\boldsymbol{G}(\boldsymbol{P}(\boldsymbol{x}, \boldsymbol{y}), Q(x, y))=
$$

$$
\begin{gathered}
\min (P(x, y)-Q(x, y), Q(x, y)-P(x, y) \\
+6), P(x, y) \geq Q(x, y) \\
\min (Q(x, y)-P(x, y), P(x, y)-Q(x, y) \\
+6), P(x, y)<Q(x, y)
\end{gathered}
$$

Where value of $\mathrm{D}$ lies between 0 and 1 . The minimum of the resulting matching distances is considered to be the final distance.

\section{EXPERIMENTAL RESULTS}

Performance of the proposed system is tested on publicly available "IIT Delhi finger knuckle database version 1.0" [9]. This database has been acquired from 158 users in IIT Delhi campus using digital camera. All the subjects in the database are in the age group 16 to 55 years. Images are in bitmap format. Resolution of these images is $80 \times 100$ pixels. Sample knuckle images are as shown in figure 1. In our proposed work we considered two knuckle image samples of same person and are assigned to the same class. More than 100 such classes are formed and considered for testing the experimental results. The proposed system is entirely implemented in Matlab 7.4.0.
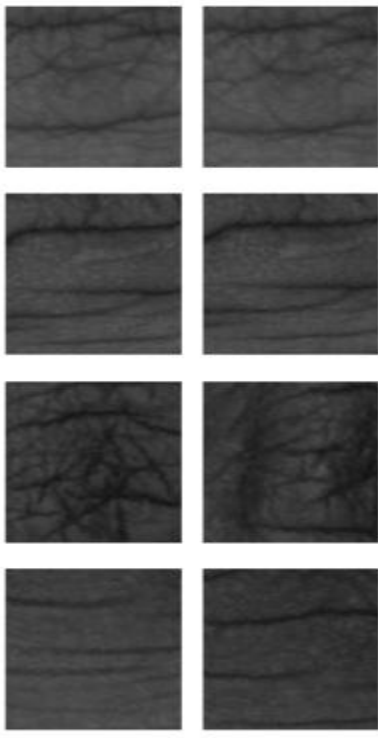

Figure 1. Sample knuckle images from database-two knuckle images per person

Experimental results of knuckle identification using Gabor filter method is as shown in figure 2. Person is identified and identification number along with minimum distance of respective person is displayed on the output screen .Figure 3 shows the features extracted from knuckle image using 2D Gabor filter. Distance plot using Gabor filter method is as shown in figure 4. Distances between test knuckle image and classes are calculated. Class with minimum distance is then identified. If this distance is greater than threshold value then the person is recognized as imposter. And if it is less than threshold value then person is recognized as genuine by giving corresponding identification number as output as shown in figure 2. To decide best threshold value testing of proposed system is carried out both on imposter and genuine knuckle images as shown in figure 4 .Figure shows the sample data of both genuine and imposter person, 10 samples for 
each. Distance is considered on $\mathrm{Y}$ axis and samples on $\mathrm{X}$ axis. Distance threshold is decided based on experiment in order to obtain better recognition rate as shown in figure 4.During the experiment distance threshold is considered as 3.5.

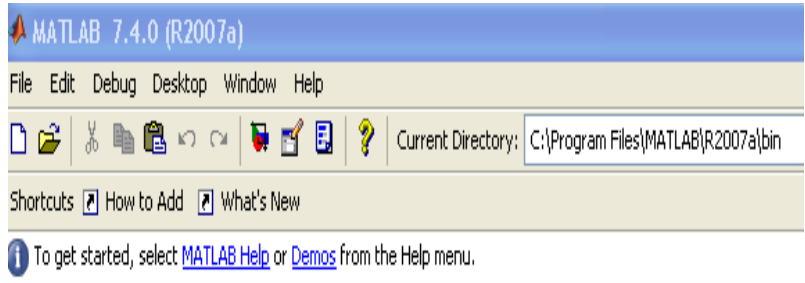

Features extraction for Finger-Knuckle-Print recognition... please wait.

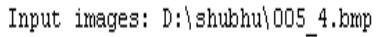

Recognized ID: 5

$$
0.3147
$$

\section{Figure 2. Result of Knuckle recognition using 2D Gabor} filter

Testing is done on Compaq Intel(R) Pentium(R) Dual core processor with $988 \mathrm{MB}$ RAM. Software is entirely implemented in Matlab 7.4.0. Computation speed of feature extraction and finger knuckle identification offered by proposed system is found satisfactory. Time required for knuckle print identification using Gabor method is recorded as $1.9 \mathrm{~ms}$.

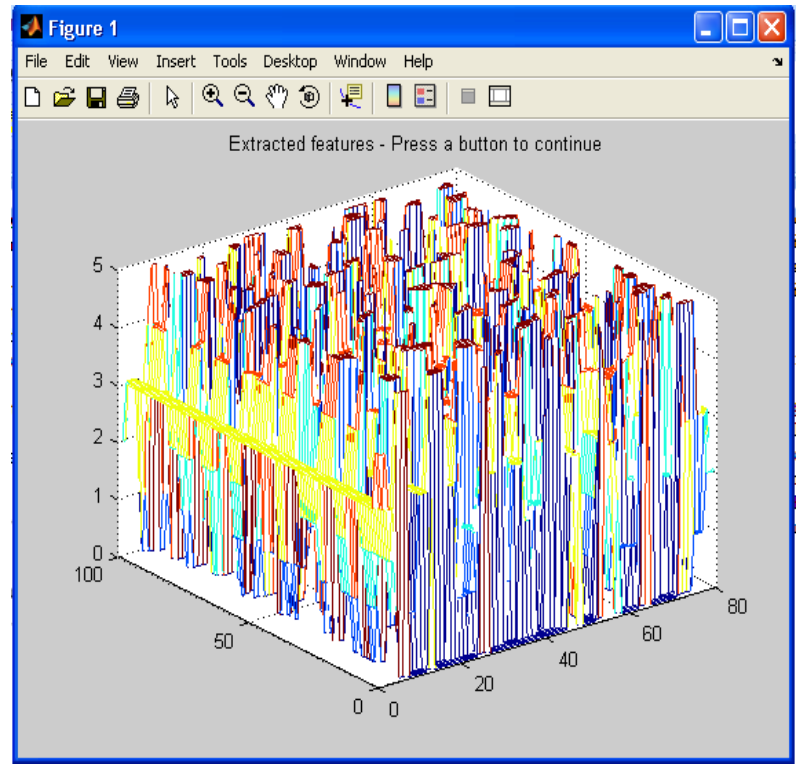

Figure3. Knuckle feature extraction using 2D Gabor filter.

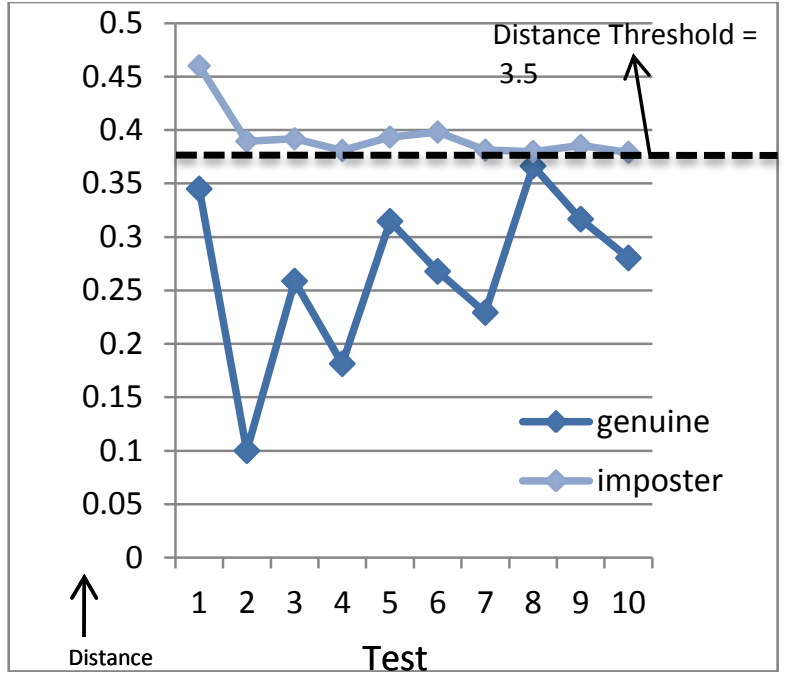

Figure 4. Distribution of imposter and genuine matching distance using Gabor filter

At given distance threshold, the probability of accepting imposter known as false acceptance rate (FAR ) and probability of rejecting genuine user is known as false rejection rate (FRR ) are obtained. Equal Error Rate ( EER ) is an optimal rate where FAR is equal to FRR. Graphically, EER is recognized as the crossing point between FAR and FRR. It is commonly used to determine the overall accuracy of the system and serve as comparative measure against the other biometric systems. Correct recognition rate (CRR) for identification is used to measure the performance of the system and is found satisfactory. CRR of the system is given as $\mathrm{CRR}=(\mathrm{X} 1 / \mathrm{X} 2) \times 100$, where $\mathrm{X} 1$ denotes the total number of correct recognitions of FKP images and $\mathrm{X} 2$ denotes the total number of persons in database. Table 1 shows performance of proposed system and other related work.

Table 1: Performance of proposed system and others

\begin{tabular}{|c|c|}
\hline Matching Technique & CRR (\%) \\
\hline Correlation Coefficient [12] & $91 \%$ \\
\hline Knuckle code [10] & $98.6 \%$ \\
\hline Angular distance [7] & $97 \%$ \\
\hline Non-Enh-SIFT[3] & $98 \%$ \\
\hline Enh-SIFT[3] & $99 \%$ \\
\hline $\begin{array}{c}\text { Non Enh -SURF[3] } \\
\text { Enh-SURF[3] }\end{array}$ & $99 \%$ \\
\hline $\begin{array}{c}\text { PCA [11] } \\
\text { Feature based Approach } \\
\text { (Gabor ) }\end{array}$ & $99 \%$ \\
\hline
\end{tabular}




\section{CONCLUSION}

This paper presented a novel approach for personal identification using two dimensional finger knuckle image. Feature based approach 2D Gabor filter method is employed for knuckle identification. It is observed that proposed feature based method for feature extraction and classification have been quite effective in achieving high performance in knuckle classification. In addition to this, the proposed Finger Knuckle Identification technique has several advantages such as user friendliness, moderate size, cost-effectiveness, etc. It has a great potential to be future improved and employed in real applications. Proposed work considered single finger knuckle image for identification. In future this can also be extended by considering multiple finger knuckle images. Finger geometry and knuckle blending information may also be combined and used for person identification. Variations in knuckle creases due to some disease may degrade the performance, this requires further investigation. In near future we may plan to test this method on large database.

\section{REFERENCES}

[1]. Jain, A.K., Flynn, P., Ross, A. (eds.): Handbook of Biometrics. Springer, Heidelberg(2007)

[2]. Kumar A and Ravikanth C, "Personal authentication using finger knuckle surface", IEEE Transactions on Information Forensics and Security, 4(1):98 -110, 2009.

[3]. Badrinath G S, Nigam A. and Gupta P, "An Efficient Finger-knuckle-print based Recognition System Fusing SIFT and SURF Matching Scores", Information and communication Security, pp374-387, 2011.[3].Woodard D.L., Flynn P.J., "Finger surface as a biometric identifier", CVIU, vol. 100, pp. 357-384, 2005.

[4]. D. Gabor, Theory of communication, Journal of the Institute of Electrical Engineers 93 (1946) 429-457.

[5]. L. Nanni, A. Lumini, On selecting Gabor features for biometric authentication, International Journal of Computer Applications in Technology 35 (1) (2009) 2328.

[6]. A. Kong, An evaluation of Gabor orientation as a feature for face recognition, in: Proceedings of ICPR'08,2008.

[7]. Zhang L, Zhang L, Zhang D, "Finger-Knuckle Print: A New Biometric Identifier", Image Processing ICIP, IEEE International Conference, pp1981-84, Nov2009.

[8]. T.S. Lee, Image representation using 2D Gabor wavelet, IEEE Trans. Pattern Analysis and Machine Intelligence18 (10) (1996) 957-971.

[9].http://www4.comp.polyu.edu.hk/ CSajaykr/IITD/iitd_knu ckle.htm

[10].Kumar A and Zhou Y, "Human identification using knuckle codes", Proceedings BTAS, Washington, 2009.
[11]. Neware S, Mehta K, Zadgaonkar A , "Finger Knuckle Identification using Principal Component Analysis and Nearest Mean Classifier", International Journal of Computer Applications (0975 - 8887) Volume 70- No.9, May 2013.

[12]. Woodard D.L., Flynn P.J., "Finger surface as a biometric identifier", CVIU, vol. 100, pp. 357-384, 2005.

[13]. Zhang L, Zhang L, Zhang D, Zhu H, “Ensemble of local and global information for finger-knuckle-print recognition", Pattern Recognition, 44(9):1990 - 1998, 2011.

[14].Shoichiro A, Koichi I, Takafumi A, "Finger-KnucklePrint Recognition Using BLPOC-Based Local Block Matching”, IEEE, 2011.

[15]. Rui Z, Tao L, Shunyan H, Jianying S, “ A Novel Approach of Personal Identification Based on the Fusion of Multifinger Knuckleprints", Advances in information Sciences and Service Sciences(AISS) Volume3, Number10,November 2011.

[16]. Kanta Ratha N, Bolle R, "Automatic Fingerprint Recognition System”, Springer, pp 17-18.

[17]. Gupta P, Rattani A, Mehrotra H, Kaushik A, "Multimodal biometrics system for efficient human Recognition", Biometric Technique for human Identification III,Proceedings of SPIE,Apr.2006.

[18]. Choras M, Kazil R, "Knuckle Biometrics Based on Texture Features", International Workshop on Emerging Techniques and Challenges for Hand-Based Biometrics (ETCHB), IEEE, 2010.

[19]. Kumar A, Zhou Y, "Personal Identification using Finger Knuckle Orientation Features", Electronics Letters, vol. 45, no. 20, September 2009.

[20].Kumar A, Ch Ravikanth, "Biometric Authentication Using Finger Back Surface", Computer Vision and Pattern Recognition, IEEE Conference, pp1-6, June2007.

[21]. Zhang L, Zhang L, Zhang D, "Finger-Knuckle-Print Verification Based on Band-Limited Phase-Only Correlation", Proceedings of the 13th International Conference on Computer Analysis of Images and Patterns, pp. 141-148,Springer, 2009.

[22].Jain A, Kumar A, "Biometrics of Next Generation: An Overview", Second Generation Biometrics, Springer, 2010.

[23]. Turk,M. and A. Pentland, Eigenfaces for Recognition. Journal of Cognitive Neuroscience, 3(1),1991,71-86

[24].Neware S, Mehta K, Zadgaonkar, "Finger Knuckle Surface Biometrics", International Journal of Emerging Technology and Advanced Engineering ,ISSN 22502459, Volume 2, Issue 12, December 2012 